\title{
Nonequilibrium thermodynamics of acoustic phonons in suspended graphene
}

\author{
Robin J. Dolleman ${ }^{1},{ }^{1, *}$ Gerard J. Verbiest $\odot,{ }^{2}$ Yaroslav M. Blanter, ${ }^{1}$ Herre S. J. van der Zant, ${ }^{1}$ and Peter G. Steeneken ${ }^{1,2,}$ \\ ${ }^{1}$ Kavli Institute of Nanoscience, Delft University of Technology, Lorentzweg 1, 2628 CJ Delft, The Netherlands \\ ${ }^{2}$ Department of Precision and Microsystems Engineering, Delft University of Technology, Mekelweg 2, 2628 CD Delft, The Netherlands
}

(Received 25 September 2019; accepted 19 February 2020; published 11 March 2020)

\begin{abstract}
Recent theory has predicted large temperature differences between the in-plane [longitudinal (LA) and transverse (TA)] and out-of-plane [flexural (ZA)] acoustic phonon baths in locally heated suspended graphene. To verify these predictions, and their implications for understanding the nonequilibrium thermodynamics of two-dimensional (2D) materials, experimental techniques are needed. Here, we present a method to determine the acoustic phonon bath temperatures from the frequency-dependent mechanical response of suspended graphene to a power-modulated laser. The mechanical motion reveals two counteracting contributions to the thermal expansion force, that are attributed to fast positive thermal expansion by the in-plane phonons and slower negative thermal expansion by the out-of-plane phonons. The magnitude of the two forces reveals that the in-plane and flexural acoustic phonons are at very different temperatures in the steady state, with typically observed values of the ratio $\Delta T_{\mathrm{LA}+\mathrm{TA}} / \Delta T_{\mathrm{ZA}}$ between 0.2 and 3.7. These deviations from the generally used local thermal equilibrium assumption $\left(\Delta T_{\mathrm{LA}+\mathrm{TA}}=\Delta T_{\mathrm{ZA}}\right)$ can affect the experimental analysis of the thermal properties of $2 \mathrm{D}$ materials.
\end{abstract}

DOI: 10.1103/PhysRevResearch.2.012058

The thermal properties of graphene [1] are unconventional, because of the large difference between its in-plane and outof-plane lattice dynamics [2-7]. Therefore, much research has focused on characterizing graphene's thermal conductivity, for example, by using Raman spectroscopy or electrical heaters [8-15]. Recent theoretical work by Vallabhaneni et al. has suggested that local optical heating of suspended graphene can lead to large temperature differences between the in-plane (longitudinal LA and transverse TA) and out-ofplane (flexural, ZA) acoustic phonon baths, which is caused by differences in the thermal conductivities of the different types of phonons, and their weak mutual interactions [16]. By using optical techniques, it has been confirmed that electrons and optical phonons can show very different temperatures compared to the acoustic phonons in two-dimensional (2D) materials [17-22], but since optical techniques are only sensitive to the population of the in-plane phonons, a strong thermal nonequilibrium between the in-plane and out-of-plane (flexural) acoustic phonon modes themselves has not been established in an experiment. Since it has been hypothesized that such a thermal nonequilibrium might impact the interpretation of the widely used Raman spectroscopy technique to measure the thermal conductivity of graphene [16], there is a need to characterize the temperatures of the in-plane and flexural acoustic phonon baths separately.

\footnotetext{
*Present address: 2nd Institute of Physics, RWTH Aachen University, 52074 Aachen, Germany; R.J.Dolleman@tudelft.nl

$\dagger$ P.G.Steeneken@tudelft.nl

Published by the American Physical Society under the terms of the Creative Commons Attribution 4.0 International license. Further distribution of this work must maintain attribution to the author(s) and the published article's title, journal citation, and DOI.
}

Recently, several optomechanical techniques to characterize the time-dependent heat transport in suspended 2D materials have been developed [23-26]. Here, we demonstrate the use of an optomechanical technique to distinguish two thermal expansion force contributions with different time constants and opposite signs. It is argued that these contributions can be attributed to the in-plane and flexural acoustic phonons. The differences in time constant and sign allow us to obtain information on the modal temperatures of the respective phonon baths.

Figure 1 shows the device and setup that is used to thermally actuate and measure the motion of suspended graphene membranes. The sample fabrication is identical to that in earlier work [23]. Single-layer graphene grown by chemical vapor deposition (CVD) is transferred over dumbbell-shaped cavities in a $\mathrm{Si} / \mathrm{SiO}_{2}$ substrate $(300 \mathrm{~nm}$ deep, various diameters) using a support polymer. The polymer is dissolved and the sample is dried using critical point drying, which breaks one of the dumbbell drums while the other side survives, resulting in a circular graphene drum with a venting channel to the environment that prevents gas from being trapped between the membrane and the substrate.

To actuate the motion, the membrane is heated in a vacuum (pressure lower than $1 \times 10^{-5}$ mbar) by a sinusoidally powermodulated blue laser. The blue laser, which is focused at the center of the drum, has a wavelength of $405 \mathrm{~nm}$, an average incident laser power of $0.36 \mathrm{~W}$, and its sinusoidal modulation amplitude is $0.24 \mathrm{~W}$. Electrons in the graphene drum are photoexcited, and decay into thermal phonons in less than a picosecond $[16,27,28]$ via electron-phonon scattering. Compared to the timescales at which phonons exchange heat $(>0.1 \mathrm{~ns})$, the power transfer from light to lattice vibrations via electron-phonon scattering can thus be considered instantaneous. The out-of-plane membrane motion is read out using a photodiode (PD) that detects the reflected intensity of a 


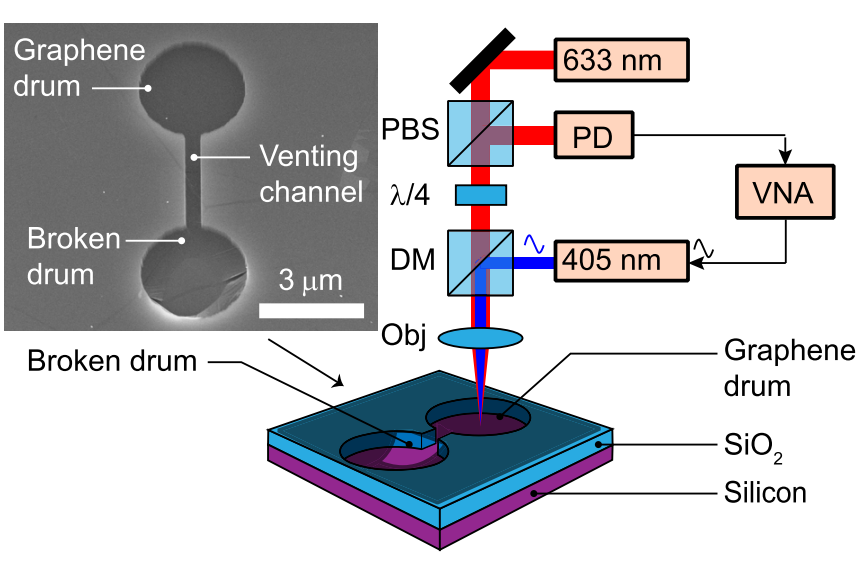

FIG. 1. Scanning electron microscope image of a typical device used in the experiment and the experimental setup to measure the thermomechanical response of suspended graphene membranes.

633-nm red helium-neon laser with a power of $1.2 \mathrm{~mW}$ focused on the center of the membrane, that is modulated by the position-dependent absorption of the graphene membrane $[29,30]$. The estimated waist diameter of the focal point is $0.67 \pm 0.1 \mu \mathrm{m}$ for the red laser and $0.57 \pm 0.1 \mu \mathrm{m}$ for the blue laser, which was calculated using Gaussian beam theory [24]. A vector network analyzer (VNA) measures the frequency-dependent amplitude and phase of the signal at the output of a photodetector relative to the modulated blue laser power. The signal is corrected for parasitic phase shifts due to delays in the optical and electronic path using a calibration measurement [23], which ensures that the voltage change from the photodiode is linearly proportional to the deflection of the membrane. All experiments are performed at room temperature.

Figures 2(a) and 2(b) show the real and imaginary amplitude of the membrane's motion as a function of frequency. To analyze the data, the membrane temperature response $\Delta T$ to a modulated input power $P_{\mathrm{ac}}$ is modeled by the heat equation $\mathcal{C} \Delta \dot{T}+\Delta T / \mathcal{R}=P_{\mathrm{ac}} e^{i \omega t}$, where $\mathcal{C}$ is the effective heat capacitance and $\mathcal{R}$ is the effective thermal resistance of the membrane. $\Delta T$ is the average temperature change over the suspended drum area with respect to the environmental temperature $T_{0}$, such that the total temperature is given by $T=T_{0}+\Delta T$. The thermal expansion force is assumed to be proportional to the change in temperature $\Delta T(t), F(t)=$ $\alpha_{\text {eff }} \Delta T(t)$. Furthermore, we assume that far below the mechanical resonance frequency the displacement amplitude $z(t)=F(t) / k$, where $k$ is the effective membrane stiffness. The time-dependent thermal expansion force represented in the frequency domain is then $[23,24]$

$$
F_{\omega} e^{i \omega t} \propto z_{\omega} e^{i \omega t}=C_{\text {slow }} \frac{e^{i \omega t}}{i \omega \tau_{\text {slow }}+1},
$$

where $C_{\text {slow }}$ is a constant, representative of the amplitude of the thermal expansion force at low frequencies, used for fitting. $\omega$ is the driving frequency, $\tau_{1}=\mathcal{R C}$ the thermal time constant, and $F_{\omega}$ is obtained by the Fourier transform of $F(t)$. The imaginary part of Eq. (1) has an extremum with amplitude $C_{\text {slow }} / 2$ at radial frequency $\omega=1 / \tau_{\text {slow }}$, as indicated in Figs. 2(a) and 2(b). Only the imaginary part of Eq. (1) is fit to the data, showing good agreement with the experimentally obtained imaginary amplitude. If the real part corresponding to this fit is plotted, however, it is found that below the resonance frequency there is an additional offset $C_{\text {fast }}$, between the real part of Eq. (1) and the measurement, that is almost frequency independent, as indicated in Figs. 2(a) and $2(\mathrm{~b})$. To quantify the value of $C_{\text {fast }}$ experimentally, the average value of the difference between the real part of the model and the experimental data [see Figs. 2(a) and 2(b)] at frequencies below the resonance frequency is taken. All drums with a negative value of $C_{\text {slow }}$ have a positive offset in the real part $C_{\text {fast }}$ and drums with a positive $C_{\text {slow }}$ have a negative $C_{\text {fast }}$ [Fig. 2(c)]. We deduce from this correlation between $C_{\text {slow }}$ and $C_{\text {fast }}$ that the offset $C_{\text {fast }}$ is not due to optical crosstalk from the blue laser [23], but related to the membrane motion, because optical crosstalk in the setup is independent of the motion of the drum and independent of the sign of $C_{\text {slow }}$.

Since the motion that corresponds to the offset $C_{\text {fast }}$ cannot be accounted for by the force in Eq. (1), it is interpreted as evidence for the existence of an additional second contribution to the thermal expansion force, with a different time constant $\tau_{\text {fast }}$. This results in a modified expression for the total thermal
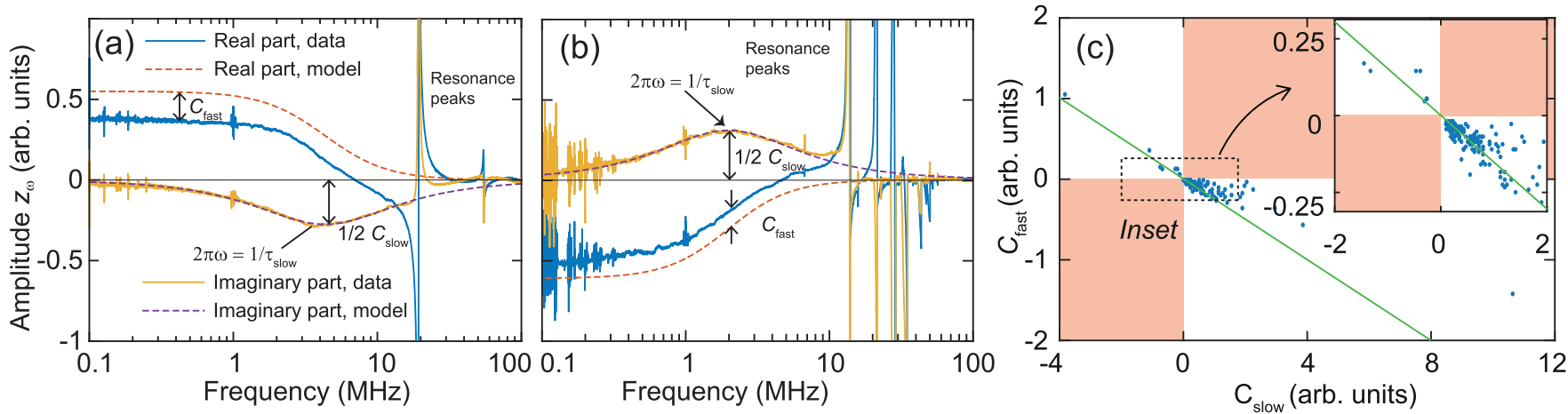

FIG. 2. Mechanical response of a suspended graphene membrane in response to an intensity-modulated laser. (a) The real and imaginary part of the amplitude of a resonator with a diameter of $4 \mu \mathrm{m}$. (b) The real and imaginary part of a 6- $\mu \mathrm{m}$-diam drum. (c) Scatter plot with the amplitude of the force $C_{\text {fast }}$ on the vertical axis and the amplitude $C_{\text {slow }}$ on the horizontal axis. Each dot represents a different device with a total of 201 devices measured. 


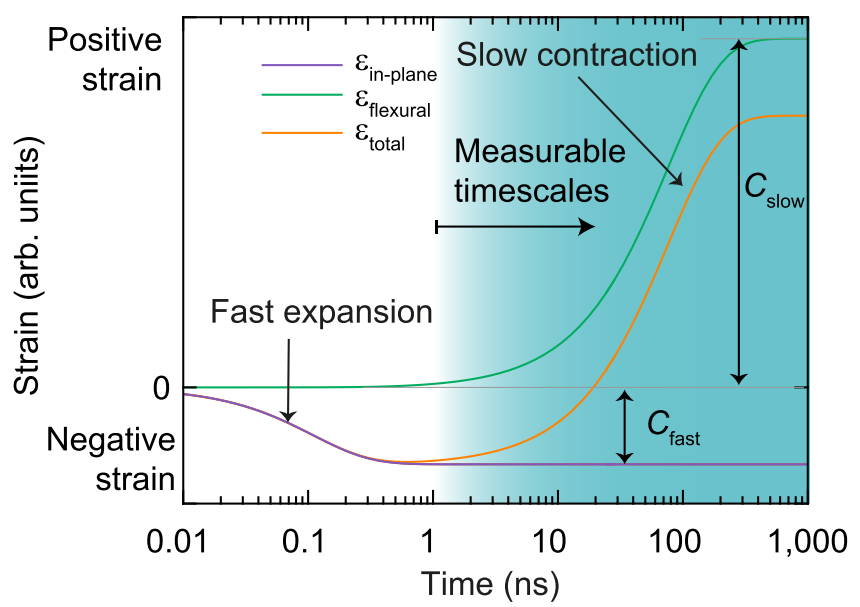

FIG. 3. Calculated time-domain representation of the strain if the laser is suddenly switched on at time $t=0 \mathrm{~s}$.

expansion force $F_{\omega}$,

$$
F_{\omega} e^{i \omega t}=C_{\text {slow }} \frac{e^{i \omega t}}{i \omega \tau_{\text {slow }}+1}+C_{\text {fast }} \frac{e^{i \omega t}}{i \omega \tau_{\text {fast }}+1} .
$$

If $\omega \tau_{\text {fast }} \ll 1$, the second contribution to the actuation force produces a constant offset in the real part and does not affect the imaginary part of $F_{\omega}$. Furthermore, a key finding of this work is that $C_{\text {fast }}$ always has an opposite sign with respect to $C_{\text {slow }}$ [Fig. 2(c)], meaning that both of these forces are counteracting at low frequencies. To show this effect more clearly, the frequency domain response of Eq. (2) is converted to a step response in the time domain in Fig. 3, using typical values of $\tau_{\text {slow }}$ found in this work and an estimate of $\tau_{\text {fast }}$ based on theory [31]. Our measurement thus indicates that when a constant heating power is suddenly applied at $t=0$, the membrane initially expands at short timescales $\tau_{\text {fast }}$ and then slowly contracts at longer timescales $\tau_{\text {slow }}$. To explain this observation, the microscopic origin of the thermal expansion contributions in graphene is analyzed in more detail.

The force that actuates the membrane $F(t)$ is directly proportional to the in-plane stress $\sigma(t)$, which is linearly related to the in-plane strain $\epsilon(t)$ by the elasticity matrix. For a membrane clamped around its circumference, this thermally induced strain is related to the internal energies of the phonons and the mechanical properties of the lattice by the equation [32]

$$
\epsilon(t)=\epsilon_{0}-\frac{1}{4 B} \sum_{j} \gamma_{j} U_{j}(t),
$$

where $\epsilon_{0}$ is the initial prestrain at the reference temperature when $\Delta T=0 \mathrm{~K}, B$ the bulk modulus, $\gamma_{j}$ the mode-dependent Grüneisen parameter, and $U_{j}(t)$ the phonon energy per unit volume for phonon mode $i$. Note that $\epsilon(t)$ is the total strain with respect to the initial positive (tensile) prestrain $\epsilon_{0}$ at a reference temperature for which $U_{j}=0$, which is reduced by thermal expansion of the membrane. Thermal expansion of the substrate is neglected in this analysis, since it absorbs less laser power than the graphene and because the volume where the heat can diffuse through is much larger, resulting in negligible temperature changes of the substrate. Only the contributions of the acoustic phonon modes are included in the following analysis since the in-plane optical phonon states are not occupied at room temperature and the flexural optical phonons have a Grüneisen parameter close to zero [33]. It is well known that the Grüneisen parameter for the flexural phonons $\gamma_{Z A}$ has a negative sign in graphene, while the Grüneisen parameter for the in-plane longitudinal acoustic $\left(\gamma_{\mathrm{LA}}\right)$ and transverse acoustic mode $\left(\gamma_{\mathrm{TA}}\right)$ is positive [32]. At low laser modulation frequencies, the internal energy $U_{j}(t)$ of all phonon modes is in phase with the blue laser, such that the sign of the thermal expansion force only depends on the sign of the Grüneisen parameter. Based on these considerations, the most likely conclusion is that the opposite signs of $C_{\text {slow }}$ and $C_{\text {fast }}$ in the experiments in Fig. 2 can be attributed to the opposite signs of the in-plane and out-of-plane phonon mode Grüneisen parameters [32]. We furthermore hypothesize that the flexural ZA phonons have a longer thermal timescale $\tau_{\text {slow }}$, because they experience a large thermal interface resistance at the edge of the drum [31], while the fast timescale $\tau_{\text {fast }}$ is attributed to the in-plane phonons. The theory that theoretically supports the correctness of this hypothesis is presented in a separate article [31].

The average internal energy $U_{j}$ of the suspended graphene is modulated by the blue laser with an amplitude that depends on the heat flux absorbed by each mode $P_{j}$, the mode's Grüneisen parameter $\gamma_{j}$, and its thermal time constant $\tau_{j}$. We find expressions for the average internal energies $U_{j}$ in the Supplemental Material [34] and substitute these in Eq. (3) to obtain

$$
\frac{C_{\text {fast }}}{C_{\text {slow }}}=-\frac{\left(\gamma_{\mathrm{LA}}+\gamma_{\mathrm{TA}}\right) P_{\mathrm{LA}+\mathrm{TA}} \tau_{\mathrm{LA}+\mathrm{TA}}}{\gamma_{\mathrm{ZA}} P_{\mathrm{ZA}} \tau_{\mathrm{ZA}}},
$$

where $\tau_{\mathrm{LA}+\mathrm{TA}}$ is the fast time constant associated with the inplane phonons (attributed to $\tau_{\text {fast }}$ for both LA and TA phonons) and $\tau_{\mathrm{ZA}}$ is the slow time constant from the flexural phonons (attributed to $\tau_{\text {slow }}$ ). Furthermore, an analytical expression for the time constants corresponding to the flexural phonons $\tau_{\mathrm{ZA}}$ is derived by taking only the interaction between the phonon modes at the boundary into account. Using the model in Ref. [31], it is found that the time constant $\tau_{\mathrm{ZA}}$ can be approximated by the expression

$$
\tau_{\mathrm{ZA}}=\frac{a}{2 \sum \bar{w}_{1 z \rightarrow 2 r} c_{\mathrm{ZA}}},
$$

where $a$ is the radius of the drum, $\sum \bar{w}_{1 z \rightarrow 2 r}$ the fraction of ZA phonons that transmit over the boundary towards the environment, and $c_{\mathrm{ZA}}$ is the $\mathrm{ZA}$ phonon propagation velocity. Both $\sum \bar{w}_{1 z \rightarrow 2 r}$ and $c_{\mathrm{ZA}}$ are tension-dependent parameters, which increase their value with increasing tension.

Based on Eq. (4), a linear relation between the parameters $-C_{\text {fast }} / C_{\text {slow }}$ and $1 / \tau_{\mathrm{ZA}}$ is expected, assuming that $\tau_{\mathrm{LA}+\mathrm{TA}}$ and $P_{\mathrm{LA}+\mathrm{TA}}$ are constant because they are relatively insensitive to tension variations. A study of their correlations is therefore useful as a test for the hypothesis behind Eq. (4), since $\tau_{\mathrm{ZA}}$ and $-C_{\text {fast }} / C_{\text {slow }}$ can be extracted independently from the measurement. Figure 4 shows a plot with $-C_{\text {fast }} / C_{\text {slow }}$ on the horizontal axis and $1 / \tau_{\text {slow }}$ on the vertical axis, for drums with a diameter of $6 \mu \mathrm{m}$. We find a significant linear correlation between $a / \tau_{\text {slow }}$ and $-C_{\text {fast }} / C_{\text {slow }}$, which is in agreement with the models underlying Eq. (4). Interestingly, in the 


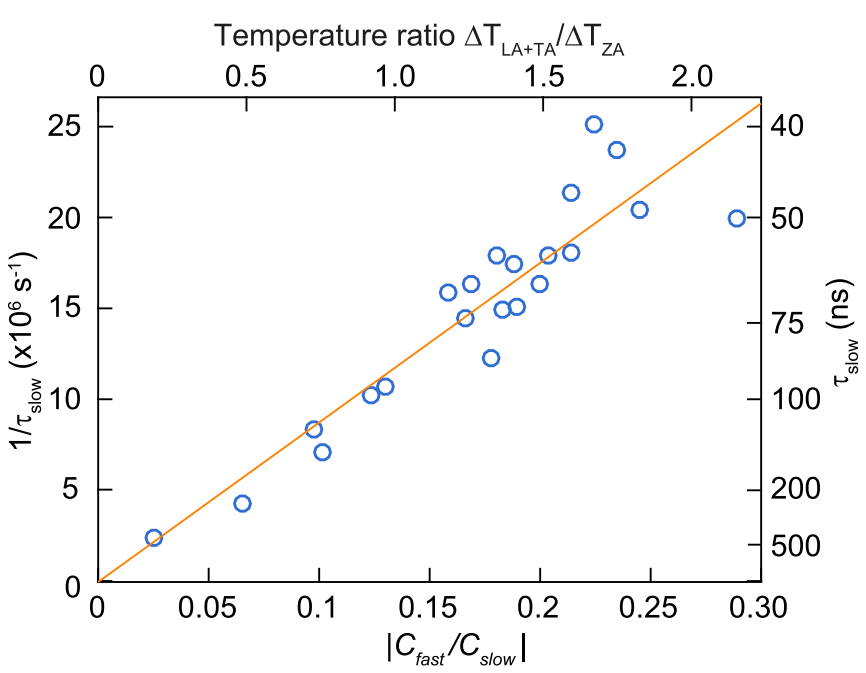

FIG. 4. Plot of $1 / \tau_{\text {slow }}$ vs $\left|C_{\text {fast }} / C_{\text {slow }}\right|$ for drums with a diameter of $6 \mu \mathrm{m}$; data sets for other diameters can be found in the Supplemental Material [34]. The second horizontal axis shows the temperature ratio calculated from Eq. (6).

Supplemental Material [34], we find that this correlation is diameter dependent.

The ratio $-C_{\text {fast }} / C_{\text {slow }}$ can be used to estimate the degree of thermal nonequilibrium in the system. It is assumed that the changes in modal temperatures of the in-plane phonons are equal $\Delta T_{\mathrm{LA}+\mathrm{TA}}=\Delta T_{\mathrm{LA}}=\Delta T_{\mathrm{TA}}$, based on the results obtained in Ref. [16]. Combining Eq. (4) with the thermal expansion term $C_{j}=\gamma_{j} \rho c_{p, j} \Delta T_{j}$, where $c_{p, j}$ is the modal specific heat at constant pressure and $\rho$ is the density, we obtain, using Eq. (4),

$$
\frac{\Delta T_{\mathrm{LA}+\mathrm{TA}}}{\Delta T_{\mathrm{ZA}}}=-\frac{C_{\text {fast }}}{C_{\text {slow }}} \frac{\gamma_{\mathrm{ZA}} c_{p, \mathrm{ZA}}}{\gamma_{\mathrm{LA}} c_{p, \mathrm{LA}}+\gamma_{\mathrm{TA}} c_{p, \mathrm{TA}}} .
$$

The temperature ratio at low frequencies is thus proportional to $-C_{\text {fast }} / C_{\text {slow }}$ with a proportionality constant that can be evaluated from theory. Using $\gamma_{\mathrm{LA}}=1.06, \gamma_{\mathrm{TA}}=0.40$, and $\gamma_{\mathrm{ZA}}=-4.17$ [33], and the modal specific heats $\left[c_{p, \mathrm{LA}}=\right.$ $\left.104 \mathrm{~J} /(\mathrm{kg} \mathrm{K}), c_{p, \mathrm{TA}}=225 \mathrm{~J} /(\mathrm{kg} \mathrm{K}), c_{p, \mathrm{ZA}}=358 \mathrm{~J} /(\mathrm{kg} \mathrm{K})\right]$ calculated at an environmental temperature of $293.15 \mathrm{~K}$, we obtain $\Delta T_{\mathrm{LA}+\mathrm{TA}} / \Delta T_{\mathrm{ZA}}=-7.45 C_{\mathrm{fast}} / C_{\text {slow }}$. Using this expression, the temperature ratio is calculated and shown on the second horizontal axis in Fig. 4.

The average value of the ratio $\Delta T_{\mathrm{LA}+\mathrm{TA}} / \Delta T_{\mathrm{ZA}}$ is of the order of 1 (see Fig. 4 and the Supplemental Material [34]). This is surprising, because the observation that $\tau_{\mathrm{LA}+\mathrm{TA}} \ll$ $\tau_{\mathrm{ZA}}$ suggests that the ZA phonons have a very low thermal conductance and therefore, according to Eqs. (4) and (6), we should expect $\Delta T_{\mathrm{ZA}} \gg \Delta T_{\mathrm{LA}+\mathrm{TA}}$. This apparent contradiction between the observed thermal time constants and the temperature ratio is explained by the selective electron-phonon coupling in graphene, which causes most of the heat supplied by the laser to end up in the LA and TA phonon bath, while the ZA phonons only receive a small fraction of this heat due to the weak coupling [16]. The small value of $\tau_{\mathrm{LA}+\mathrm{TA}} / \tau_{\mathrm{ZA}}$ in Eq. (4) is thus partially compensated by the large value of $P_{\mathrm{LA}+\mathrm{TA}} / P_{\mathrm{ZA}}$, thereby causing the temperature of the in-plane and flexural acoustic phonon bath to be in the same order of magnitude.

In Fig. 4, although a few drums have almost the same value for in-plane and out-of-plane temperature, in most drums large variations in the temperature ratio are observed, with $\Delta T_{\mathrm{LA}+\mathrm{TA}} / \Delta T_{\mathrm{ZA}}$ varying from 0.2 to 2.2 . This provides evidence for the existence of a strong nonequilibrium thermal state. According to Eq. (5), $\tau_{\mathrm{ZA}}$ is tension dependent, while $\tau_{\mathrm{LA}+\mathrm{TA}}$ is not expected to be tension dependent. Consequently, according to Eq. (4), a linear correlation between $\tau_{\text {slow }}$ and $\left|C_{\text {fast }} / C_{\text {slow }}\right|$ as found in Fig. 4 shows that the large variations in the temperature ratio are dominated by device-to-device variations in the pretension via its effect on the thermal time constant $\tau_{\mathrm{ZA}}$. Similar large variations in $\tau_{\mathrm{ZA}}$ have been observed in our previous work [23]. Some devices deviate from this linear correlation (see Supplemental Material [34]), and this might suggest that other effects such as wrinkles and other imperfections are also playing a role in the variations in the temperature ratio. In the calculations by Sullivan $e t$ al. [22], a ratio $\Delta T_{\mathrm{LA}+\mathrm{TA}} / \Delta T_{\mathrm{ZA}}=2.9$ was obtained. Most of the samples in this work show a temperature ratio that is considerably lower than predicted by Sullivan et al. This may be attributed to effects of sidewall adhesion and tension in the membrane, which are not taken into account in existing theoretical works. In Ref. [31], we worked out a detailed model including these effects and find that this can explain the disparity in the measured values of $\Delta T_{\mathrm{LA}+\mathrm{TA}} / \Delta T_{\mathrm{ZA}}$.

The observed nonequilibrium effect is important for the interpretation of heat transport in graphene, because it suggests that a full understanding of thermal conductivity will require unraveling the individual phonon mode contributions, which is still an open challenge [5]. Sullivan et al. [22] experimentally studied the population of optical phonon modes with respect to the acoustic LA phonon mode, however, experimental methods and reports on the effect of nonequilibrium between different acoustic phonon modes on the thermal conductance are lacking. The present work shows that this nonequilibrium condition can be quantified by exploiting the difference in the sign of the thermal expansion in graphene. With the presented methodology, the current work demonstrates the importance of the large velocity differences and weak interactions between acoustic phonons for the heat transport in graphene, and shows that the classical heat equation, with a single thermal conductivity, cannot be used because the thermal conductance by in-plane and out-of-plane phonons is significantly different. This affects any suspended graphene device that is locally heated, due to the inherent selective electron-phonon coupling and weak interaction between the phonon modes

Other two-dimensional materials are expected to show similar effects as observed in this work if they exhibit a weak mode interaction and a large negative Grüneisen parameter for the flexural acoustic phonons. This might hold for other monatomic two-dimensional materials at room temperature [32], but also transition-metal dichalcogenides such as $\mathrm{MoS}_{2}, \mathrm{MoSe}_{2}$, and $\mathrm{WS}_{2}$ at low temperatures $(<100 \mathrm{~K})$ [35].

To conclude, we analyzed the frequency-dependent mechanical motion of graphene membranes which are heated by a modulated laser. The analysis shows a clear correlation between the magnitude and sign of the thermal expansion 
parameters $C_{\text {fast }}$ and $C_{\text {slow }}$ [Fig. 2(c)]. Interestingly, $C_{\text {fast }}$ and $C_{\text {slow }}$ have opposite signs, which provides evidence that the fast thermal expansion parameter (that was not measured before) is of opposite sign than the slow thermal expansion parameter in graphene. Further analysis shows that a second correlation exists between the peak frequency $1 / \tau_{\text {slow }}$ and the ratio $C_{\text {fast }} / C_{\text {slow }}$ (Fig. 4). This correlation is in line with models for the in-plane and out-of-plane acoustic phonons and provides qualitative evidence that $C_{\text {fast }}$ can be attributed to the in-plane LA and TA branch, whereas $C_{\text {slow }}$ can be attributed to the out-of-plane ZA branch. The results show that the sign of the thermal expansion coefficient in graphene membranes is time dependent (Fig. 3). The presented analysis might prove useful to analyze the thermal properties in a range of other ultrathin materials.

The authors thank Applied Nanolayers B.V. for supply and transfer of the single-layer graphene. We furthermore thank C. Stampfer, J. Sonntag, and J. E. Sader for fruitful discussions. This work is part of the research programme Integrated Graphene Pressure Sensors (IGPS) with Project No. 13307 which is financed by the Netherlands Organisation for Scientific Research (NWO). The research leading to these results also received funding from the European Union's Horizon 2020 research and innovation programme under Grant Agreement No. 785219 Graphene Flagship.
[1] A. K. Geim and K. S. Novoselov, The rise of graphene, Nat. Mater. 6, 183 (2007).

[2] A. A. Balandin, Thermal properties of graphene and nanostructured carbon materials, Nat. Mater. 10, 569 (2011).

[3] D. L. Nika and A. A. Balandin, Two-dimensional phonon transport in graphene, J. Phys.: Condens. Matter 24, 233203 (2012).

[4] E. Pop, V. Varshney, and A. K. Roy, Thermal properties of graphene: Fundamentals and applications, MRS Bull. 37, 1273 (2012).

[5] D. L. Nika and A. A. Balandin, Phonons and thermal transport in graphene and graphene-based materials, Rep. Prog. Phys. 80, 036502 (2017).

[6] X. Xu, J. Chen, and B. Li, Phonon thermal conduction in novel 2D materials, J. Phys.: Condens. Matter 28, 483001 (2016).

[7] X. Gu, Y. Wei, X. Yin, B. Li, and R. Yang, Colloquium: Phononic thermal properties of two-dimensional materials, Rev. Mod. Phys. 90, 041002 (2018).

[8] A. A. Balandin, S. Ghosh, W. Bao, I. Calizo, D. Teweldebrhan, F. Miao, and C. N. Lau, Superior thermal conductivity of singlelayer graphene, Nano Lett. 8, 902 (2008).

[9] S. Chen, Q. Li, Q. Zhang, Y. Qu, H. Ji, R. S. Ruoff, and W. Cai, Thermal conductivity measurements of suspended graphene with and without wrinkles by micro-Raman mapping, Nanotechnology 23, 365701 (2012).

[10] W. Cai, A. L. Moore, Y. Zhu, X. Li, S. Chen, L. Shi, and R. S. Ruoff, Thermal transport in suspended and supported monolayer graphene grown by chemical vapor deposition, Nano Lett. 10, 1645 (2010).

[11] S. Ghosh, W. Bao, D. L. Nika, S. Subrina, E. P. Pokatilov, C. N. Lau, and A. A. Balandin, Dimensional crossover of thermal transport in few-layer graphene, Nat. Mater. 9, 555 (2010).

[12] J. H. Seol, I. Jo, A. L. Moore, L. Lindsay, Z. H. Aitken, M. T. Pettes, X. Li, Z. Yao, R. Huang, D. Broido, N Mingo, R. S. Ruoff, and L. Shi, Two-dimensional phonon transport in supported graphene, Science 328, 213 (2010).

[13] J.-U. Lee, D. Yoon, H. Kim, S. W. Lee, and H. Cheong, Thermal conductivity of suspended pristine graphene measured by Raman spectroscopy, Phys. Rev. B 83, 081419(R) (2011).

[14] X. Xu, L. F. C. Pereira, Yu. Wang, J. Wu, K. Zhang, X. Zhao, S. Bae, C. Tinh Bui, R. Xie, J. T. L. Thong, B. H. Hong, K. P.
Loh, D. Donadio, B. Li, and B. Özyilmaz, Length-dependent thermal conductivity in suspended single-layer graphene, Nat. Commun. 5, 3689 (2014).

[15] C. Faugeras, B. Faugeras, M. Orlita, M. Potemski, R. R. Nair, and A. K. Geim, Thermal conductivity of graphene in Corbino membrane geometry, ACS Nano 4, 1889 (2010).

[16] A. K. Vallabhaneni, D. Singh, H. Bao, J. Murthy, and X. Ruan, Reliability of Raman measurements of thermal conductivity of single-layer graphene due to selective electron-phonon coupling: A first-principles study, Phys. Rev. B 93, 125432 (2016).

[17] H. Wang, J. H. Strait, P. A. George, S. Shivaraman, V. B. Shields, M. Chandrashekhar, J. Hwang, F. Rana, M. G. Spencer, C. S. Ruiz-Vargas, and J. Park, Ultrafast relaxation dynamics of hot optical phonons in graphene, Appl. Phys. Lett. 96, 081917 (2010).

[18] Y. D. Kim, H. Kim, Y. Cho, J. H. Ryoo, C.-H. Park, P. Kim, Y. S. Kim, S. Lee, Y. Li, S.-N. Park et al., Bright visible light emission from graphene, Nat. Nanotechnol. 10, 676 (2015).

[19] D.-H. Chae, B. Krauss, K. von Klitzing, and J. H Smet, Hot phonons in an electrically biased graphene constriction, Nano Lett. 10, 466 (2009).

[20] S. Berciaud, M. Y. Han, K. F. Mak, L. E. Brus, P. Kim, and T. F. Heinz, Electron and Optical Phonon Temperatures in Electrically Biased Graphene, Phys. Rev. Lett. 104, 227401 (2010).

[21] A. Block, M. Liebel, R. Yu, M. Spector, Y. Sivan, F. J. G de Abajo, and N. F. van Hulst, Tracking ultrafast hot-electron diffusion in space and time by ultrafast thermomodulation microscopy, Sci. Adv. 5, eaav8965 (2019).

[22] S. Sullivan, A. Vallabhaneni, I. Kholmanov, X. Ruan, J. Murthy, and L. Shi, Optical generation and detection of local nonequilibrium phonons in suspended graphene, Nano Lett. 17, 2049 (2017).

[23] R. J. Dolleman, S. Houri, D. Davidovikj, S. J. CartamilBueno, Y. M. Blanter, H. S. J. van der Zant, and P. G. Steeneken, Optomechanics for thermal characterization of suspended graphene, Phys. Rev. B 96, 165421 (2017).

[24] R. J. Dolleman, D. Lloyd, M. Lee, J. S. Bunch, H. S. J. van der Zant, and P. G. Steeneken, Transient thermal characterization 
of suspended monolayer $\mathrm{MoS}_{2}$, Phys. Rev. Materials 2, 114008 (2018).

[25] N. Morell, S. Tepsic, A. Reserbat-Plantey, A. Cepellotti, M. Manca, I. Epstein, A. Isacsson, X. Marie, F. Mauri, and A. Bachtold, Optomechanical measurement of thermal transport in two-dimensional $\mathrm{MoSe}_{2}$ lattices, Nano Lett. 19, 3143 (2019).

[26] A. Blaikie, D. Miller, and B. J. Alemán, A fast and sensitive room-temperature graphene nanomechanical bolometer, Nat. Commun. 10, 4726 (2019).

[27] J. M. Dawlaty, S. Shivaraman, M. Chandrashekhar, F. Rana, and M. G. Spencer, Measurement of ultrafast carrier dynamics in epitaxial graphene, Appl. Phys. Lett. 92, 042116 (2008).

[28] D. Singh, J. Y. Murthy, and T. S. Fisher, Spectral phonon conduction and dominant scattering pathways in graphene, J. Appl. Phys. 110, 094312 (2011).

[29] A. Castellanos-Gomez, R. van Leeuwen, M. Buscema, H. S. J. van der Zant, G. A. Steele, and W. J. Venstra, Singlelayer $\mathrm{MoS}_{2}$ mechanical resonators, Adv. Mater. 25, 6719 (2013).
[30] R. J. Dolleman, D. Davidovikj, H. S. J. van der Zant, and P. G. Steeneken, Amplitude calibration of 2D mechanical resonators by nonlinear optical transduction, Appl. Phys. Lett. 111, 253104 (2017).

[31] R. J. Dolleman, Y. M. Blanter, H. S. J. van der Zant, P. G. Steeneken, and G. J. Verbiest, Phonon scattering at kinks in suspended graphene, Phys. Rev. B 101, 115411 (2020).

[32] X.-J. Ge, K.-L. Yao, and J.-T. Lü, Comparative study of phonon spectrum and thermal expansion of graphene, silicene, germanene, and blue phosphorene, Phys. Rev. B 94, 165433 (2016).

[33] S. Mann, R. Kumar, and V. K. Jindal, Negative thermal expansion of pure and doped graphene, RSC Adv. 7, 22378 (2017).

[34] See Supplemental Material at http://link.aps.org/supplemental/ 10.1103/PhysRevResearch.2.012058 for additional data on the diameter dependence and the derivation of the expression for the ratio $C_{\text {fast }} / C_{\text {slow }}$.

[35] B. Peng, H. Zhang, H. Shao, Y. Xu, X. Zhang, and H. Zhu, Thermal conductivity of monolayer $\mathrm{MoS}_{2}, \mathrm{MoSe}_{2}$, and $\mathrm{WS}_{2}$ : Interplay of mass effect, interatomic bonding and anharmonicity, RSC Adv. 6, 5767 (2016). 